\title{
Gender differences of relationship between serum lipid indices and type 2 diabetes mellitus: a cross-sectional survey in Chinese elderly adults
}

\author{
Xiao-Wei Ji ${ }^{1,2 \#}$, Guo-Shan Feng ${ }^{1,2 \#}$, Hong-Lan $\mathrm{Li}^{2}$, Jie Fang ${ }^{2}$, Jing Wang ${ }^{2}$, Qiu-Ming Shen ${ }^{2}$ Li-Hua Han ${ }^{2}$, \\ Da-Ke Liu ${ }^{2}$, Yong-Bing Xiang ${ }^{1,2}$ \\ ${ }^{1}$ School of Public Health, Shanghai Jiao Tong University School of Medicine, Shanghai, China; ${ }^{2}$ State Key Laboratory of Oncogene and Related \\ Genes \& Department of Epidemiology, Shanghai Cancer Institute, Renji Hospital, Shanghai Jiao Tong University School of Medicine, Shanghai, \\ China \\ Contributions: (I) Conception and design: YB Xiang; (II) Administrative support: All authors; (III) Provision of study materials or patients: All authors; \\ (IV) Collection and assembly of data: All authors; (V) Data analysis and interpretation: XW Ji, GS Feng, HL Li, YB Xiang; (VI) Manuscript writing: \\ All authors; (VII) Final approval of manuscript: All authors. \\ \#These authors contributed equally to this work. \\ Correspondence to: Yong-Bing Xiang, MD, MSc. State Key Laboratory of Oncogene and Related Genes \& Department of Epidemiology, Shanghai \\ Cancer Institute, Renji Hospital, Shanghai Jiao Tong University School of Medicine, No. 25, Lane 2200, Xie Tu Road, Shanghai 200032, China. \\ Email: ybxiang@shsci.org.
}

Background: To investigate the gender differences of the relationships between clinical serum lipid indices and type 2 diabetes mellitus (T2DM) in Chinese elderly adults.

Methods: Between 2014 and 2016, participants selected from three communities in an urban district of Shanghai were measured for serum lipid indices of low-density lipoprotein cholesterol (LDL-c), highdensity lipoprotein cholesterol (HDL-c), total cholesterol (TC), and triglyceride (TG). Age and multivariate adjusted logistic regression models were utilized to estimate the odds ratios (ORs) and 95\% confidence intervals (CIs) of serum lipid indices on T2DM prevalence.

Results: In total, 4,023 male and 3,862 female participants were included in this study, with the T2DM prevalence proportions of $13.03 \%$ and $11.73 \%$, respectively. In association analysis, the serum levels of LDL-c, HDL-c, TC were significant between non-T2DM individuals and T2DM patients in men, but the HDL-c and TG in women. LDL-c/HDL-c, TG/HDL-c, and TC/HDL-c ratios were associated with the T2DM prevalence only in women. In the multivariate analysis, a higher serum LDL-c level was positively associated with a reduced risk of T2DM prevalence in men with OR (95\% CI) of $0.57(0.39-0.85)(\mathrm{P}=0.006)$. Higher ratios of LDL-c/HDL-c, TG/HDL-c, and TC/HDL-c were all more likely associated with the decreased risks of T2DM prevalence with the ORs ranging from 0.45 to 0.62 in men (all $\mathrm{P}<0.05$ ), but not in women.

Conclusions: High LDL-c concentration was significantly associated with a lower T2DM prevalence in men. A gender difference of the associations between the lipid ratios and T2DM prevalence was observed for LDL-c/HDL-c and TC/HDL-c ratios, which might be validated in female T2DM prevalence in the future.

Keywords: Serum lipid indices; type 2 diabetes mellitus (T2DM); cross-sectional study; gender difference

Submitted Mar 13, 2020. Accepted for publication Sep 30, 2020.

doi: $10.21037 / \mathrm{atm}-20-2478$

View this article at: http://dx.doi.org/10.21037/atm-20-2478 


\section{Introduction}

Diabetes mellitus is a global health issue that describes a series of metabolic disorders characterized by high blood glucose levels. As released by the International Diabetes Federation (IDF) in 2018, 451 million people (aging 18 to 99 years old) have been estimated with diabetes worldwide in 2017 (1). However, the numbers are 108 million in 1980 (2), 171 million in 2000 (3), and 415 million in 2015 (4), which show an obvious ascending trend. Such a great number of diabetes patients burden the world with high medical care costs, increase mortality, and reduce the quality of life. These relative healthcare issues and burdens are not only caused by type 2 diabetes mellitus (T2DM) itself, but also by other organs' problems induced by high glucose, such as cardiovascular diseases (CVDs), retinopathy, kidney and brain problems (5). China has the world's largest number of diabetes patients. As estimated by a large and nationally representative survey of Chinese adults in 2013, about 109 million adults in total in mainland China suffered from diabetes (with a prevalence of $10.9 \%)(6)$.

One of the main features of T2DM is diabetic dyslipidemia, which makes effects on $72-85 \%$ of T2DM patients (7). The exact mechanisms of diabetic lipoprotein abnormalities have not been fully understood. One of the most accepted explanations is that dyslipidemia is caused by quantitative and qualitative changes (8). The quantitative change means the increase and decrease of the lipoprotein cholesterol concentrations, which has ever been explored and reviewed (9). For the qualitative alteration, it means the size and density alterations of lipoprotein cholesterol particles with the capability to attribute to lipid abnormalities. The main quantitative abnormalities of lipoprotein include the increase of triglyceride (TG) and low-density lipoprotein cholesterol (LDL-c), as well as the reduction of high-density lipoprotein cholesterol (HDL-c) (10). A Chinese cohort study published recently indicates that TG is the strongest predictor for the onset of diabetes, and besides that, one standard deviation (SD) increase of body mass index (BMI) will elevate the risk of 1.29 times compared to people who have normal glucose tolerance and prediabetes (11). Another cohort study notes that the increased serum levels of total cholesterol (TC), TG, TC/HDL-c, and TG/HDL-c ratios may increase the risk of T2DM incidence (12). Despite the quantitative alteration, researchers also suggest that the "quality" of LDL-c may exert greater influence on T2DM for the identification potential of dense LDL-c to people with higher risks of T2DM (13). The mechanisms of lipoprotein size contributing to the pathogenesis of T2DM may be attributed to the modulation of inflammatory responses that impair beta-cell function and beta-cell mass (14).

Mounting evidence of gender-specific effects on T2DM has been raised (15), and the inclusion of the gender dimension in biomedical research has been called for by many organizations (16). Differences in genetic background, life behaviors, and environmental factors contribute to the discrepancy of T2DM and its associated complications in different genders. European men are usually diagnosed with diabetes at a younger age and with a lower BMI than women (17). Sex hormone modulates glucose and lipid homeostasis in central and peripheral targets. As reported before, higher levels of androgens lead to increased body weight in women (18); relatively higher testosterone levels in women and lower levels in men are related to incident diabetes (19). Socioeconomic status, which usually assesses by educational level, income, and occupation, are also found to play different roles in men and women (20). Health behaviors, such as smoking, alcohol consumption, and tea drinking, also have gender disparities on T2DM (21-23). All the above evidence implied that gender imbalances exist in T2DM. In our study, we aim to evaluate the relationship between clinical lipid metabolism indices and T2DM prevalence in a community-based study in urban Shanghai, which is located in the east of China. From this cross-sectional study, we firstly provide the epidemiological evidence to validate the relationship between clinical lipid indices and T2DM prevalence modifying by gender. These results may then help to identify subgroups of the population who are at a high risk of T2DM. In terms of public health, it will also provide recommendations for people who have lipid metabolic abnormalities to have medical interventions as early as possible. We present the following article in accordance with the STROBE reporting checklist (available at http://dx.doi.org/10.21037/atm-20-2478).

\section{Methods}

\section{Study populations \& settings}

In this study, participants who measured serum lipid indexes were selected from three communities in an urban district in Shanghai between 2014 and 2016. A total of 3,862 female and 4,023 male participants were enrolled 
from two cohort studies, which started in 1997 and 2002, separately $(24,25)$. The variables from the baseline and follow-up surveys were chosen to be exposure factors. All the questionnaire surveys and the anthropometric measurements were collected by trained interviewers who were retired and professional medical workers. The study was performed according to the guidelines of the Declaration of Helsinki 2013 (26) and approved by the Renji Hospital Ethics Committee of Shanghai Jiao Tong University School of Medicine (KY2019-196), and written informed consent was obtained from each participant before the interview.

\section{Clinical measurements, laboratory analysis \& definitions}

Blood samples were collected after overnight fasting of at least 10 hours at the checkup in the community health centers. Measurements had been done while the participants were on their usual diet and took no medications. Fasting LDL-c, HDL-c, TG, and TC were measured by the standardized enzymatic colorimetric methods. All the clinical assays were done by the Shanghai Lan Wei Medical Laboratory Company Limited in the Changing District of Shanghai using Roche reagents.

The outcomes of T2DM were defined as undergoing treatment with insulin or oral hypoglycemic agents (antidiabetes drug usage), or the concentration of fast blood glucose was $\geq 7.0 \mathrm{mmol} / \mathrm{L}$, or the participant had a personal history of T2DM (27). T2DM was diagnosed by experts from the community-health centers or hospitals with rich clinical and pathological experience. Participants' ages were calculated as the check date of the clinical testing minus the date of birth. Baseline BMI was calculated as weight $(\mathrm{kg}) / \mathrm{height}^{2}\left(\mathrm{~m}^{2}\right)$ and utilized in analysis instead of followup BMI in order to minimize the bias of the weight change after T2DM diagnosis. Other variables, such as education (classified into four categories, primary school and below, junior high school, senior high school, and tertiary and above), occupation (three categories, classified into professional, clerical, and manual workers), menopause (only for women, yes/no), smoking (yes/no), alcohol drinking (yes/no), tea drinking (yes/no), medical history of coronary heart disease (CHD, yes/no), anti-diabetes drug usage (yes/ no), and anti-hypertension drug usage (yes/no) were taken into consideration. Four clinical lipid metabolism indices were measured and categorized into normal and abnormal groups by the standard clinical concentrations. The criteria for LDL-c, TG, and TC were 3.37, 2.26, and $5.20 \mathrm{mmol} / \mathrm{L}$, separately (28). For HDL-c, the criteria were $1.04 \mathrm{mmol} /$ $\mathrm{L}$ for men and $1.15 \mathrm{mmol} / \mathrm{L}$ for women, respectively, which was recommended by the Shanghai Lan Wei Medical Laboratory Company Limited and the local communitybased hospitals. Continuous alternations of lipid profiles were also analyzed. In terms of the ratios of LDL-c/HDL-c, TG/HDL-c, and TC/HDL-c, we further categorized them into tertiles in non-T2DM individuals with the lowest tertile serving as the reference group, the detailed cutoff values of the tertiles in each gender were shown in Table S1.

\section{Statistical analysis}

All analyses were separately performed in each gender. Continuous variables were presented as mean \pm SD. Categorical variables were presented as frequency and percentage. Student's $t$-test was used for independent samples for comparisons of variables with normal distribution and the Wilcoxon test was used for comparisons of non-parametric ones. Chi-square tests (or Fisher's exact tests when appropriate) were used to compare the distributions of categorical variables. To figure out the relationships of LDL-c/HDL-c, TG/HDL-c, and TC/ HDL-c ratios with T2DM prevalence, logistic regression models were carried out to obtain odds ratios (ORs) and $95 \%$ confidence intervals (CIs) by calibrating all potential confounders. The regression models could be defined as two types: (I) adjusted for age (continuous scale); (II) adjusted for multivariate including age (continuous scale), education, occupation, BMI (continuous scale), personal history of CHD, menopause (only for women), antidiabetes drug usage, anti-hypertension drug usage, as well as lifestyle factors of smoking, alcohol drinking, and tea drinking.

Data analyses were conducted using SAS version 9.2 (SAS Institute, Cary, NC, USA), and re-verified by SPSS statistical package (version 20.0, SPSS Inc., USA). All statistical tests were two-sided, and a $\mathrm{P}<0.05$ was considered statistically significant.

\section{Results}

\section{Characteristics of the participants}

The demographic and basic characteristics of the participants were presented in Table 1. In total, 524 male and 453 female participants were diagnosed with T2DM, with the prevalence proportions of $13.03 \%$ and $11.73 \%$, 
Table 1 Characteristics of study participants in the cross-sectional survey in urban Shanghai

\begin{tabular}{|c|c|c|c|c|c|c|}
\hline Variables & \multicolumn{3}{|c|}{ Male } & \multicolumn{3}{|c|}{ Female } \\
\hline \multicolumn{7}{|l|}{ Continuous variables } \\
\hline Age & $66.22 \pm 7.91$ & $67.24 \pm 7.96$ & 0.006 & $65.04 \pm 8.34$ & $68.11 \pm 8.22$ & $<0.0001$ \\
\hline BMI & $23.89 \pm 2.96$ & $25.45 \pm 2.81$ & $<0.0001$ & $23.81 \pm 3.15$ & $26.23 \pm 3.30$ & $<0.0001$ \\
\hline DBP & $82.95 \pm 10.28$ & $85.22 \pm 10.54$ & $<0.0001$ & $77.06 \pm 10.02$ & $81.32 \pm 9.88$ & $<0.0001$ \\
\hline \multicolumn{7}{|l|}{ Lipid profiles } \\
\hline LDL-C & $3.12 \pm 0.88$ & $2.86 \pm 0.87$ & $<0.0001$ & $3.47 \pm 0.87$ & $3.51 \pm 1.05$ & 0.534 \\
\hline HDL-C & $1.31 \pm 0.35$ & $1.24 \pm 0.32$ & 0.0001 & $1.58 \pm 0.40$ & $1.49 \pm 0.37$ & $<0.0001$ \\
\hline LDL-c/HDL-c & $2.53 \pm 0.91$ & $2.45 \pm 0.97$ & 0.113 & $2.33 \pm 0.81$ & $2.46 \pm 0.86$ & 0.003 \\
\hline TG/HDL-c & $1.44 \pm 1.79$ & $1.69 \pm 2.10$ & 0.034 & $1.12 \pm 0.94$ & $1.45 \pm 1.81$ & 0.001 \\
\hline TC/HDL-c & $4.00 \pm 1.20$ & $4.00 \pm 1.31$ & 0.981 & $3.73 \pm 1.06$ & $3.97 \pm 1.16$ & $<0.0001$ \\
\hline \multicolumn{7}{|l|}{ Categorical variables } \\
\hline Education & & & 0.004 & & & $<0.0001$ \\
\hline$\leq$ Primary school & $189(5.41)$ & $47(8.97)$ & & $526(15.43)$ & $123(27.15)$ & \\
\hline Junior high school & 1,395 (39.95) & $221(42.18)$ & & $1,518(44.54)$ & $194(42.83)$ & \\
\hline Senior high School & $1,211(34.68)$ & $166(31.68)$ & & 1,022 (29.99) & 91 (20.09) & \\
\hline Ever smoking & $2,364(67.56)$ & $359(68.51)$ & 0.665 & $55(1.61)$ & $15(3.31)$ & 0.011 \\
\hline Ever alcohol drinking & $1,218(34.81)$ & $163(31.11)$ & 0.096 & $72(2.11)$ & $14(3.09)$ & 0.185 \\
\hline Ever tea drinking & $2,280(65.16)$ & $362(68.08)$ & 0.078 & $1,028(29.98)$ & $150(34.44)$ & 0.053 \\
\hline Hypertension & 1,392 (39.78) & $299(57.06)$ & $<0.0001$ & $1,269(37.22)$ & $280(61.81)$ & $<0.0001$ \\
\hline Anti-hypertension drug usage & $1,188(33.95)$ & $255(48.66)$ & $<0.0001$ & $930(27.28)$ & $199(43.93)$ & $<0.0001$ \\
\hline Coronary heart disease & $126(3.60)$ & $35(6.68)$ & 0.001 & $186(5.46)$ & $62(13.69)$ & $<0.0001$ \\
\hline
\end{tabular}

BMI, body mass index; SBP, systolic blood pressure; DBP, diastolic blood pressure; LDL-c, low-density lipoprotein cholesterol; HDL-c, high-density lipoprotein cholesterol; TG, Triglyceride; TC, total cholesterol; T2DM, type 2 diabetes mellitus.

respectively. The numbers of patients who had ever had anti-diabetes drug treatment were $282(53.82 \%)$ in men and $237(52.32 \%)$ in women. The estimated means of the time period from diagnosis to blood testing in T2DM prevalent patients were 7.74 years in men and 8.50 years in women, respectively. All participants were more than 50 years old. Compared to non-T2DM participants, T2DM patients were older, had higher BMI, systolic blood pressure 
Table 2 Correlations between clinical serum lipid indices and T2DM prevalence

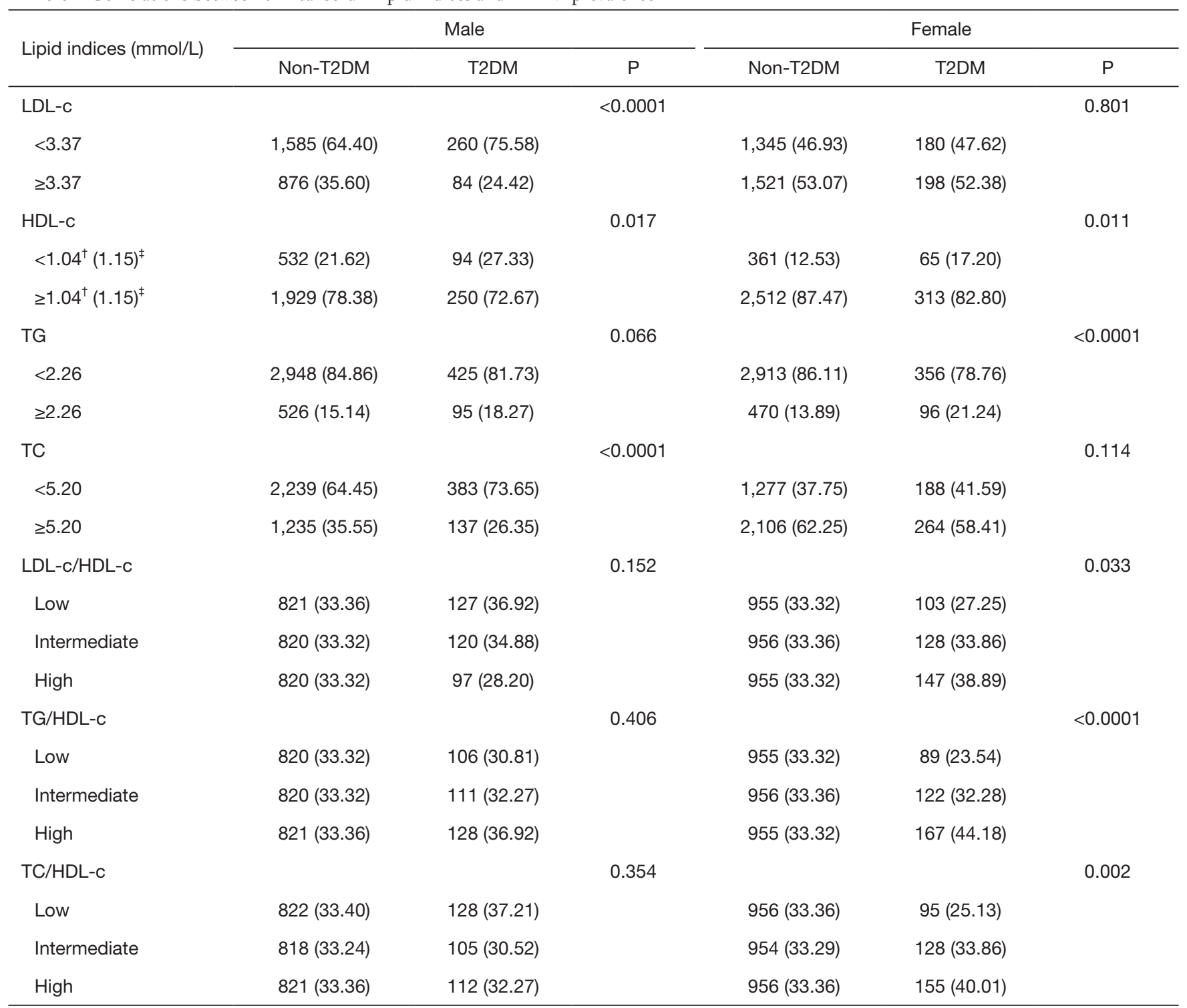

Data are expressed as $\mathrm{n}(\%) .{ }^{\dagger}$, the normal medical reference range for males; ${ }^{\ddagger}$, the normal medical reference range for females. LDL-c, low-density lipoprotein cholesterol; HDL-c, high-density lipoprotein cholesterol; TG, Triglyceride; TC, total cholesterol; T2DM, type 2 diabetes mellitus.

(SBP), and diastolic blood pressure (DBP) in both genders. Significant differences could be observed in education levels between T2DM patients and non-T2DM individuals in each gender. Referring to lifestyle factors (include smoking, alcohol drinking, and tea drinking habits), only smoking in women could be significantly observed between T2DM and non-T2DM individuals $(\mathrm{P}=0.011)$. Personal medical histories of hypertension and CHD were also significant between these two groups.

\section{Associations between clinical lipid indices and T2DM prevalence}

The associations between clinical lipid measures and T2DM prevalence stratified by gender were presented in Table 2 . In males, LDL-c, HDL-c, and TC were significantly related to T2DM prevalence; and in females, HDL-c and TG were found to be associate with T2DM prevalence. As lots of studies pinpointed that LDL-c/HDL-c, TG/HDL-c, and TC/HDL-c ratios could be served as indicators for 
T2DM incidence. We also evaluated their roles in T2DM prevalence and found no differences in males but significant meanings in females (all $\mathrm{P}<0.05$ ). Quantitative distributions of all the clinical lipid indices between T2DM cases and non-T2DM individuals also had been explored (Table 1).

\section{Logistic regression analysis between clinical lipid indices and T2DM prevalence}

Table 3 presented the relations of different lipid indices and T2DM prevalence by using the logistic regression models in separate gender. When adjusting by age, significant associations of lipid indices and T2DM prevalence were observed in higher HDL-c with ORs (95\% CIs) of 0.72 $(0.56-0.93)(\mathrm{P}=0.012)$ in men and $0.71(0.53-0.95)(\mathrm{P}=0.022)$ in women, respectively; and in higher TG concentrations with ORs (95\% CIs) of $1.09(1.03-1.15)(\mathrm{P}=0.031)$ in men and $1.26(1.16-1.37)(\mathrm{P}=0.0002)$ in women, respectively. Whereas, significant associations between LDL-c and TC and T2DM prevalence were both observed in males, but not in females. After calibrating by multivariate, null associations were detected in HDL-c, TG, or TC categories with T2DM prevalence in both genders, but significance was found in higher LDL-c concentration and the decreased risk of T2DM prevalence in males with $\mathrm{OR}$ (95\% CI) of $0.57(0.39-0.85)(\mathrm{P}=0.006)$.

We also estimated the association between the continuous change of blood lipid indices and T2DM prevalence. In the age-adjusted model, per unit alterations of lipid indices that significantly decreased T2DM prevalence were LDL-c, HDL-c, and TC in males, and it's HDL-c in females. Per unit change of TG was associated with increased risks of T2DM prevalence in both sexes with ORs (95\% CIs) of $1.09(1.03-1.15)(\mathrm{P}=0.003)$ in males and $1.26(1.16-1.37)(\mathrm{P}<0.0001)$ in females, respectively. When adjusting by multivariate factors, a unit change of LDL-c and TC were significantly associated with T2DM prevalence in males with ORs (95\% CIs) of $0.71(0.58-0.87)$ $(\mathrm{P}=0.001)$ and $0.85(0.73-0.98)(\mathrm{P}=0.021)$, respectively; no significance was found in females.

Referring to the lipid ratio indices, diverse results were detected in different genders. As shown in Table 4, all the highest levels of lipid ratios (include LDL-c/HDL-c, TG/ HDL-c, and TC/HDL-c ratios) were positively associated with higher T2DM prevalence only in females with the ORs ranging from 1.44 to $1.70(\mathrm{P}<0.01)$, but null associations were detected in males by using the age-adjusted model. Nevertheless, in multivariate models, the results shifted, meaningful associations for all higher lipid ratios with the decreased risks of T2DM prevalence were observed in men with the ORs ranging from 0.45 to 0.62 (all $\mathrm{P}<0.05$ ), but not in women. When estimating their relationships by analyzing the continuous alterations of lipid ratio indices in the multivariate model, one unit changes of LDL-c/HDL-c and TC/HDL-c were positively associated with the reduced risks of $\mathrm{T} 2 \mathrm{DM}$ prevalence.

\section{Discussion}

In our cross-sectional study, the prevalence proportions of $\mathrm{T} 2 \mathrm{DM}$ were $13.03 \%$ in men and $11.73 \%$ in women, respectively, which were a bit higher than the estimated prevalence from a national survey with $9.9 \%$ in men and $11.6 \%$ in women (29). This discrepancy might be attributed to multiple reasons. Firstly, it might be partly caused by the region factor. All participants in this study were recruited from an urban district of Shanghai City. As previous studies indicated, the prevalence rate of T2DM in urban areas was significantly higher than that in rural areas in China (29-31), which was consistent with our results. Secondly, significant differences in T2DM prevalence in different age groups of each gender were detected in our study. As some studies pointed out that age was a certified risk factor for T2DM $(31,32)$. Age variability between our study ( $\geq 50$ years) and the meta-analysis (20-75 years) (29) could also explain the reason for the relatively higher T2DM prevalence in our population. Besides, age might not be the only factor associated with high T2DM prevalence, synergistic effects contributed by other factors might also play important roles. As Table 1 presented, the education distributions of T2DM patients among participants were meaningful in both genders (Table 1 , all $\mathrm{P}<0.004$ ), which suggested education was an important variable that related to T2DM prevalence in urban Shanghai. We also found that T2DM patients had higher BMI levels than healthy participants in both genders, which were consistent with previous researches $(33,34)$. When exploring the relationships between lifestyle factors (including smoking, alcohol drinking, and tea drinking habits) and T2DM prevalence in men, no significance was detected, which was different from the meta-analyses conducting in Japan (35). In women, significant associations were detected in non-smokers and ever-smokers. As validated by a Chinese population-based study, environmental tobacco exposure increased T2DM risks with a dose-response relationship (36), which also verified our results laterally. 
Table 3 Logistic regression analysis between serum lipid indices and T2DM prevalence

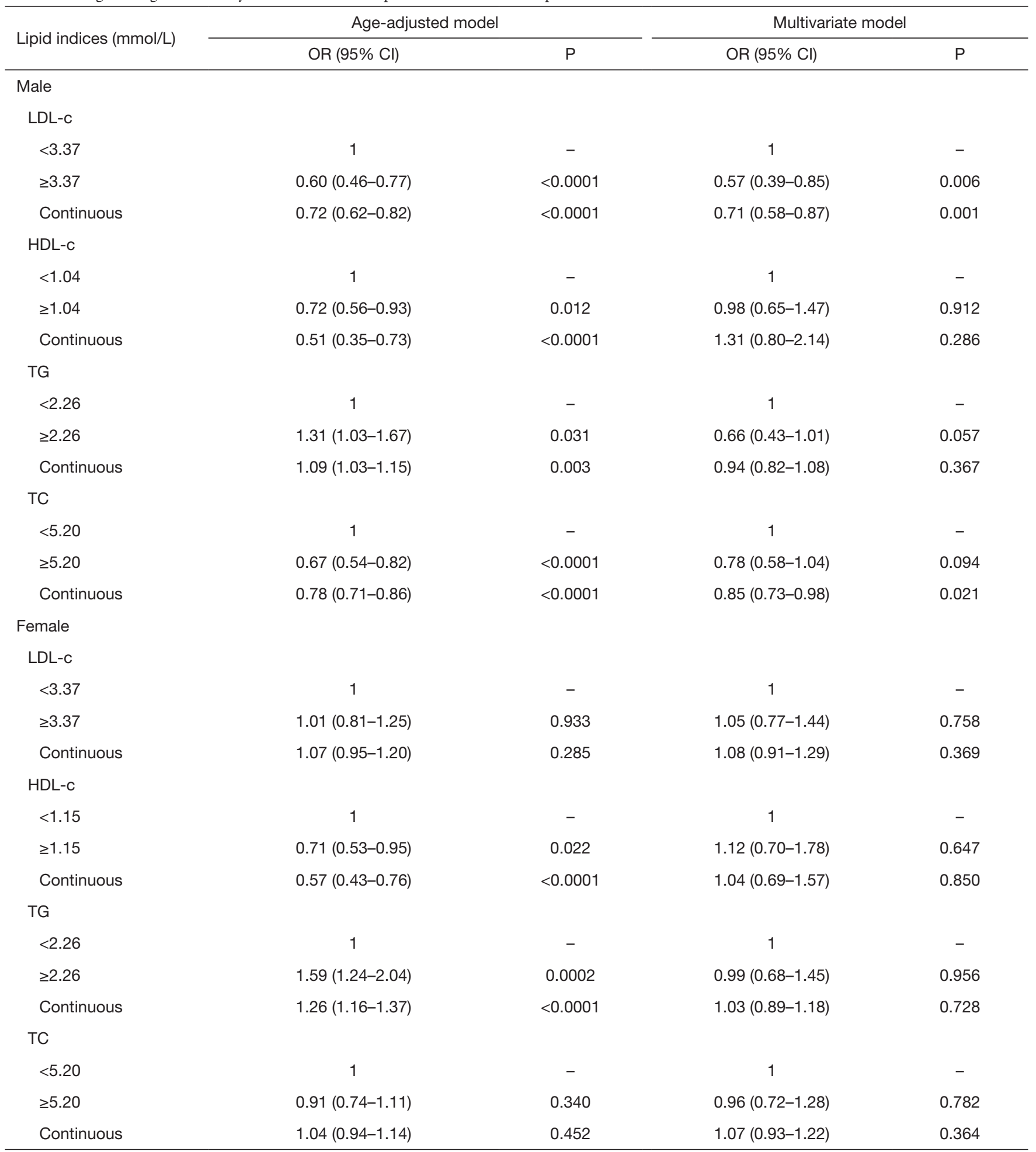

LDL-c, low-density lipoprotein cholesterol; HDL-c, high-density lipoprotein cholesterol; TG, Triglyceride; TC, total cholesterol; OR, odds ratio; $\mathrm{Cl}$, confidence interval. Age-adjusted model: adjusted by age; Multivariate model: adjusted by age (continuous), BMI (continuous), education, occupation, menopause (yes/no, only for women), smoking (yes/no), alcohol drinking (yes/no), tea drinking (yes/no), antidiabetes and anti-hypertension drug usages, and coronary heart disease (yes/no). 
Table 4 Logistic regression analysis between LDL-c/HDL-c, TG/HDL-c, TC/HDL-c ratios and T2DM prevalence

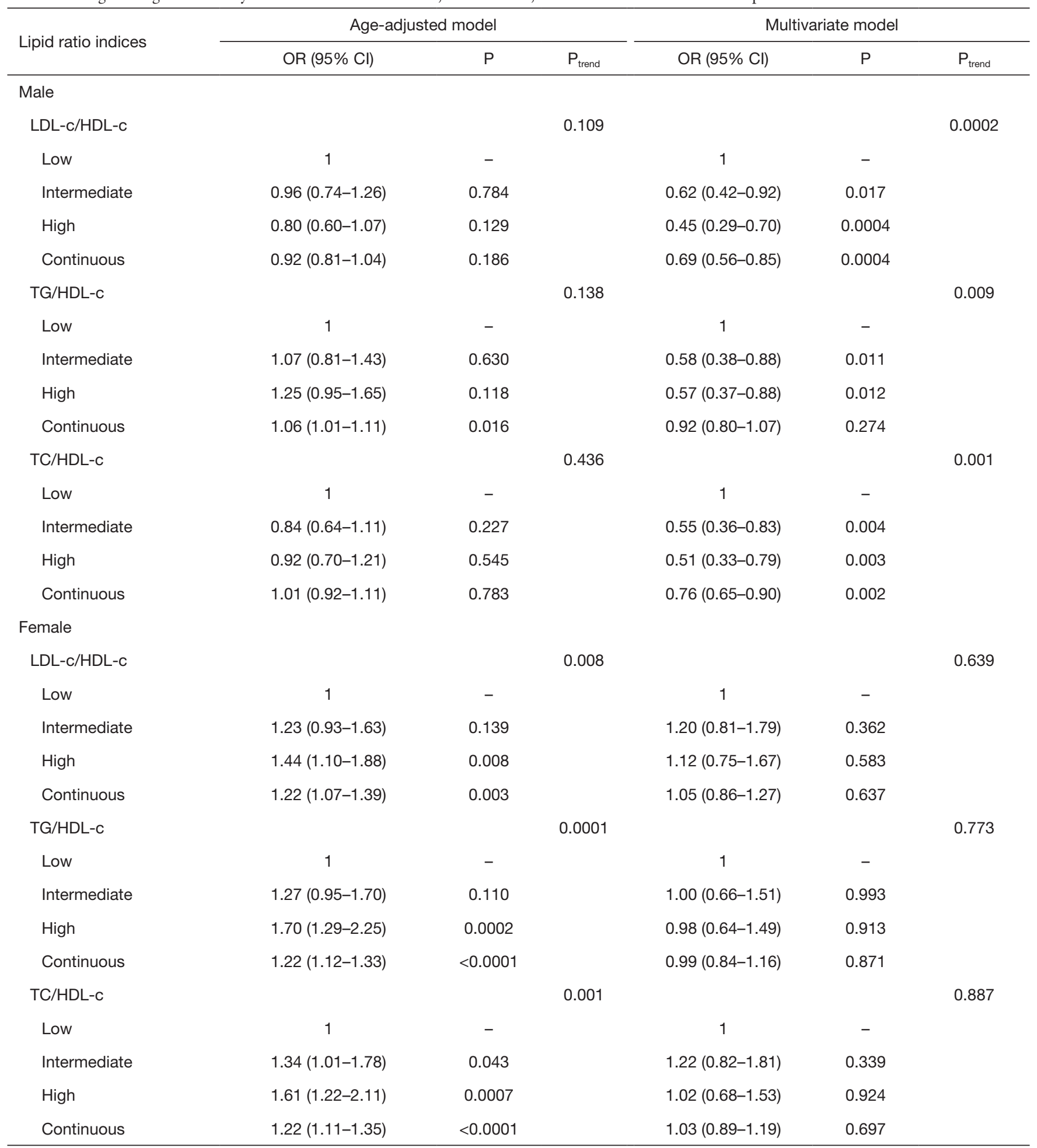

Age-adjusted model: adjusted by age; Multivariate model: adjusted by age (continuous), BMl (continuous), education, occupation, menopause (yes/no, only for women), smoking (yes/no), alcohol drinking (yes/no), tea drinking (yes/no), anti-diabetes and antihypertension drug usages, and coronary heart disease (yes/no). LDL-c, low-density lipoprotein cholesterol; HDL-c, high-density lipoprotein cholesterol; TG, Triglyceride; TC, total cholesterol; OR, odds ratio; $\mathrm{Cl}$, confidence interval. 
Higher HDL-c and lower TG levels were associated with lower risks of T2DM prevalence in both genders in our research (Table 2), which were consistent with other studies $(37,38)$. A nationwide population-based study in Korea enrolling 5,114,735 adults with a median follow-up period of 5.1 years had found that lower means of HDL-C were associated with higher risks of diabetes incidence in a stepwise manner (39). Genetic studies were also conducted to explore the relationship between HDL-c and T2DM. As indicated by a large data set with 34,840 T2DM cases and 114,982 control subjects using 140 lipid-associated genetic variants to assess the relationship between lipid profiles and T2DM, genetically determined lower HDL-c concentrations were associated with T2DM prevalence (40). Otherwise, lipid genotype scores of well-established single nucleotide polymorphism in 95 loci showed that low HDL-c or high TG levels were related to elevated risks of T2DM prevalence (41). From the above previously published studies, it could be concluded that high HDL-c levels were good for health and positively associated with the reduced risks of diabetes incidence or prevalence.

However, the roles of LDL-c and TC on diabetes prevalence had not been consistently verified in our study. In the multivariate logistic model within our research (Table 3), a higher LDL-c concentration was associated with a lower risk of diabetes prevalence in males with OR $(95 \% \mathrm{CI})$ of $0.57(0.39-0.85)(\mathrm{P}=0.006)$, but null associations were detected in females. The positive effects of LDL-c to T2DM in our study were similar to the results obtained from the Framingham Heart Study when adjusting by multivariate including sex, as it concluded that a higher LDL-c concentration was associated with a lower risk of diabetes with OR (95\% CI) of $0.81(0.70-0.93)(\mathrm{P}=0.004)(42)$. Another large community-based cross-sectional study in the Netherlands also suggested that T2DM prevalence among patients with familial hypercholesterolemia who suffering from more severe LDL mutations was significantly lower than among unaffected relatives, which also agreed with our results (43). However, contrary conclusions of higher LDL-c concentrations associated with higher risks of T2DM incidence had also been demonstrated in other studies (44). This discrepancy might be introduced by race and ethnicity, which had been recommended to be taken into consideration in clinical care (45). Interestingly, in another community-based cross-sectional study without gender-specific analysis in North China, per-SD increases of LDL-c, TG, TC, TG/HDL-c, and TC/HDL-c were all meaningfully associated with increased T2DM prevalence in the multivariate logistic model (all ORs >1.50) (46), which were totally different from ours. These disparities also suggested that race and ethnicity might not be the major influencing factor. A Mendelian random analysis (47) reported that LDL-c played a causal role in T2DM with the elevated LDL-c significantly increased the risk of T2DM, which made the effects of LDL-c on T2DM confusing compared to our results.

After exploring the effects of LDL-c/HDL-c, TG/ HDL-c, and TC/HDL-c ratios on T2DM prevalence, we found that they all had negative associations in males but positive relations in females when adjusting by age. However, their roles shifted when adjusting for multiple confounding factors in the multivariate logistic model (as shown in Table 4, higher LDL-c/HDL-c, TG/HDL-c, and TC/HDL-c ratios were associated with T2DM prevalence in males, but null associations were detected in females). This disparity might be caused by several reasons. Firstly, the variations in specific gender might be attributed to the different characteristics of two studied groups, such as their education levels, smoking habit, BMI, anti-DM drug usage, and anti-hypertension drug usage, etc., the disparities of those factors might lighten their relevance with T2DM. On the other hand, these results also implied that the strong relationships of personal characters and T2DM in separate gender (17,19-23). More importantly, the inconsistent influences of high LDL-c/HDL-c, TG/HDL-c, and TC/ HDL-c ratios in each gender neither adjusting with age nor multivariate suggested the gender-specific roles of clinical lipid indices on T2DM. Although limited gender-specific results of T2MD prevalence from other studies had been explored currently, the gender-specific effects of LDL-c on T2DM risks had been estimated. In the Bogalusa Heart Study, higher LDL-c placed girls at a disproportionally higher risk of T2DM as women (48), which suggested that LDL-c in women posed more risk effects on T2DM and implied the positive role of men, which was quite consistent with our results. As indicated by the CCMR-3B Study, the inability of women to achieve LDL-c goals was greater than men, which also implied the existence of gender imbalances in lipid metabolism (49). And another study examining lipid management also pointed out that disparities existed in lipid control in different genders (50).

In summary, no consistent conclusions could be drawn and the disparities of diverse viewpoints presented in different studies implied the necessity of further verification. Moreover, some limitations could be addressed in our study. For this study is a cross-sectional design, the relationships 
between clinical lipid indices and T2DM prevalence might be biased by other residual confounders, such as BMI. After diagnosing with T2DM, people might introduce some healthy diet habits into their lives, which would result in a decrease of BMI; and the decreased BMI would also affect T2DM in turn. Furthermore, selection bias might exist in the study, which was the drawback of all crosssectional studies. To minimize it, age balanced between our study and its original cohorts. The validations in larger population-based case-control or cohort studies with more demographic and clinical or treatment information should be recommended in the future.

\section{Conclusions}

Our study provided more information for Chinese community-based cross-sectional studies. Summarily, we enrolled 7,885 participants in total, and primarily evaluated the gender-specific relationship between serum clinical lipid indexes and T2DM in Chinese elderly adults. As similar to the results reported by other studies, low TG and high HDL-c levels were associated with decreased T2DM prevalence in both genders in our study. However, different conclusions could be drawn between other studies and ours when taking account of the roles of LDL-c and TC, which might need further explorations. Besides that, we also did a secondary analysis of the primary data and found that LDL-c/HDL-c, TG/HDL-c, and TC/HDL-c ratios had gender-specific differences between T2DM patients and non-T2DM subjects, which had rarely been done by other studies before.

\section{Acknowledgments}

We express deep gratitude to all the staff of the communitybased hospitals for their contributions to the data collection. Funding: This study was supported by the Research Grant from the State Key Laboratory of Oncogenes and Related Genes (\#91-1708). The authors have no other relevant affiliations or financial involvement with any organization or entity with a financial interest in or financial conflict with the subject matter or materials discussed in the manuscript apart from those disclosed.

\section{Footnote}

Reporting Checklist: The authors have completed the STROBE reporting checklist. Available at http://dx.doi. org/10.21037/atm-20-2478

Data Sharing Statement: Available at http://dx.doi. org/10.21037/atm-20-2478

Conflicts of Interest: All authors have completed the ICMJE uniform disclosure form (available at http://dx.doi. org/10.21037/atm-20-2478). Dr. YBX reports grants from Research Grant from the State Key Laboratory of Oncogenes and Related Genes (\#91-1708), during the conduct of the study. The other authors have no conflicts of interest to declare.

Ethical Statement: The authors are accountable for all aspects of the work in ensuring that questions related to the accuracy or integrity of any part of the work are appropriately investigated and resolved. The study was conducted in accordance with the Declaration of Helsinki (as revised in 2013). The study was approved by the Renji Hospital Ethics Committee of Shanghai Jiao Tong University School of Medicine (KY2019-196) and written informed consent was obtained from each participant before the interview.

Open Access Statement: This is an Open Access article distributed in accordance with the Creative Commons Attribution-NonCommercial-NoDerivs 4.0 International License (CC BY-NC-ND 4.0), which permits the noncommercial replication and distribution of the article with the strict proviso that no changes or edits are made and the original work is properly cited (including links to both the formal publication through the relevant DOI and the license). See: https://creativecommons.org/licenses/by-nc-nd/4.0/.

\section{References}

1. Cho NH, Shaw JE, Karuranga S, et al. IDF Diabetes Atlas: Global estimates of diabetes prevalence for 2017 and projections for 2045. Diabetes Res Clin Pract 2018;138:271-81.

2. NCD Risk Factor Collaboration. Worldwide trends in diabetes since 1980: a pooled analysis of 751 populationbased studies with 4.4 million participants. Lancet 2016;387:1513-30.

3. Wild S, Roglic G, Green A, et al. Global prevalence of diabetes: estimates for the year 2000 and projections for 2030. Diabetes Care 2004;27:1047-53.

4. Ogurtsova K, da Rocha Fernandes JD, Huang Y, et al. 
IDF Diabetes Atlas: Global estimates for the prevalence of diabetes for 2015 and 2040. Diabetes Res Clin Pract 2017;128:40-50.

5. Zheng Y, Ley SH, Hu FB. Global aetiology and epidemiology of type 2 diabetes mellitus and its complications. Nat Rev Endocrinol 2018;14;88-98.

6. Wang L, Gao P, Zhang M, et al. Prevalence and ethnic pattern of diabetes and prediabetes in China in 2013. JAMA 2017;317:2515-23.

7. Vergès B. Pathophysiology of diabetic dyslipidaemia: where are we? Diabetologia 2015;58:886-99.

8. Jialal I, Singh G. Management of diabetic dyslipidemia: An update. World J Diabetes 2019;10:280-90.

9. Hermans MP, Valensi P. Elevated triglycerides and low high-density lipoprotein cholesterol level as marker of very high risk in type 2 diabetes. Curr Opin Endocrinol Diabetes Obes 2018;25:118-29.

10. Farbstein D, Levy AP. HDL dysfunction in diabetes: causes and possible treatments. Expert Rev Cardiovasc Ther 2012;10:353-61.

11. Liu L, Guan X, Yuan Z, et al. Different contributions of dyslipidemia and obesity to the natural history of type 2 diabetes: 3-Year cohort study in China. J Diabetes Res 2019;2019:4328975.

12. Zhang M, Zhou J, Liu Y, et al. Risk of type 2 diabetes mellitus associated with plasma lipid levels: The rural Chinese cohort study. Diabetes Res Clin Pract 2018;135:150-7.

13. Rizzo M, Rini GB, Berneis K. The clinical relevance of LDL size and subclasses modulation in patients with type-2 diabetes. Exp Clin Endocrinol Diabetes 2007;115:477-82.

14. Kruit JK, Brunham LR, Verchere CB, et al. HDL and LDL cholesterol significantly influence beta-cell function in type 2 diabetes mellitus. Curr Opin Lipidol 2010;21:178-85.

15. Kautzky-Willer A, Harreiter J, Pacini G. Sex and Gender Differences in Risk, Pathophysiology and Complications of Type 2 Diabetes Mellitus. Endocr Rev 2016;37:278-316.

16. Schiebinger L, Klinge I, Sánchez de Madariaga I, et al. Gendered innovations in science, health, medicine, engineering and environment. 2011- 2015. Available online: http://genderedinnovations.stanford.edu

17. Logue J, Walker JJ, Colhoun HM, et al. Do men develop type 2 diabetes at lower body mass indices than women? Diabetologia 2011;54:3003-6.

18. Elbers JM, Asscheman H, Seidell JC, et al. Long-term testosterone administration increases visceral fat in female to male transsexuals. J Clin Endocrinol Metab 1997;82:2044-7.

19. Ding EL, Song Y, Malik VS, et al. Sex differences of endogenous sex hormones and risk of type 2 diabetes: a systematic review and meta-analysis. JAMA 2006;295:1288-99.

20. Rathmann W, Haastert B, Icks A, et al. Sex differences in the associations of socioeconomic status with undiagnosed diabetes mellitus and impaired glucose tolerance in the elderly population: the KORA Survey 2000. Eur J Public Health 2005;15:627-33.

21. Pan A, Wang Y, Talaei M, et al. Relation of active, passive, and quitting smoking with incident type 2 diabetes: a systematic review and meta-analysis. Lancet Diabetes Endocrinol 2015;3:958-67.

22. Schrieks IC, Heil AL, Hendriks HF, et al. The effect of alcohol consumption on insulin sensitivity and glycemic status: a systematic review and meta-analysis of intervention studies. Diabetes Care 2015;38:723-32.

23. Hirata A, Ohnaka K, Tashiro N, et al. Effect modification of green tea on the association between rice intake and the risk of diabetes mellitus: a prospective study in Japanese men and women. Asia Pac J Clin Nutr 2017;26:545-55.

24. Zheng W, Chow WH, Yang G, et al. The Shanghai Women's Health Study: rationale, study design, and baseline characteristics. Am J Epidemiol 2005;162:1123-31.

25. Shu XO, Li H, Yang G, et al. Cohort profile: the Shanghai Men's Health Study. Int J Epidemiol 2015;44:810-8.

26. World Medical Association. World Medical Association Declaration of Helsinki: ethical principles for medical research involving human subjects. JAMA 2013;310:2191-4.

27. Yang $W$, Lu J, Weng J, et al. Prevalence of diabetes among men and women in China. N Engl J Med 2010;362:1090-101.

28. Joint committee for developing Chinese guidelines on prevention and treatment of dyslipidemia in adults. Chinese guidelines on prevention and treatment of dyslipidemia in adults. Chin J Cardiol 2007;35:390-419.

29. Yang L, Shao J, Bian Y, et al. Prevalence of type 2 diabetes mellitus among inland residents in China (2000-2014): A meta-analysis. J Diabetes Investig 2016;7:845-52.

30. Singh RB, Bajaj S, Niaz MA, et al. Prevalence of type 2 diabetes mellitus and risk of hypertension and coronary artery disease in rural and urban population with low rates of obesity. Int J Cardiol 1998;66:65-72.

31. Han C, Zhang M, Luo X, et al. Secular trends in the 
prevalence of type 2 diabetes in adults in China from 1995 to 2014: A meta-analysis. J Diabetes 2017;9:450-61.

32. Chew BH, Ghazali SS, Ismail M, et al. Age $\geq 60$ years was an independent risk factor for diabetes-related complications despite good control of cardiovascular risk factors in patients with type 2 diabetes mellitus. Exp Gerontol 2013;48:485-91.

33. Kim JH, Noh J, Choi JW, et al. Association of Education and Smoking Status on Risk of Diabetes Mellitus: A population-based nationwide cross-sectional study. Int J Environ Res Public Health 2017;14:E655.

34. Lv J, Yu C, Guo Y, et al. Adherence to a healthy lifestyle and the risk of type 2 diabetes in Chinese adults. Int J Epidemiol 2017;46:1410-20.

35. Akter S, Goto A, Mizoue T. Smoking and the risk of type 2 diabetes in Japan: A systematic review and meta-analysis. J Epidemiol 2017;27:553-61.

36. Huang C, Chen G, Zhang M, et al. Association between environmental tobacco smoke exposure and risk of type 2 diabetes mellitus in Chinese female never smokers: A population-based cohort study. J Diabetes 2020;12:339-46.

37. Lee JS, Chang PY, Zhang Y, et al. Triglyceride and HDL-C dyslipidemia and risks of coronary heart disease and ischemic stroke by glycemic dysregulation status: the Strong Heart Study. Diabetes Care 2017;40:529-37.

38. Song S, Lee JE. Dietary patterns related to triglyceride and high-density lipoprotein cholesterol and the Incidence of type 2 diabetes in Korean men and women. Nutrients 2018;11:E8.

39. Lee SH, Kim HS, Park YM, et al. HDL-cholesterol, its variability and the risk of diabetes: a nationwide population-based study. J Clin Endocrinol Metab 2019;104:5633-41.

40. Fall T, Xie $W$, Poon $W$, et al. Using genetic variants to assess the relationship between circulating lipids and type 2 diabetes. Diabetes 2015;64:2676-84.

41. Qi Q, Liang L, Doria A, et al. Genetic predisposition to

Cite this article as: Ji XW, Feng GS, Li HL, Fang J, Wang J, Shen QM, Han LH, Liu DK, Xiang YB. Gender differences of relationship between serum lipid indices and type 2 diabetes mellitus: a cross-sectional survey in Chinese elderly adults. Ann Transl Med 2021;9(2):115. doi: 10.21037/atm-20-2478 dyslipidemia and type 2 diabetes risk in two prospective cohorts. Diabetes 2012;61:745-52.

42. Andersson C, Lyass A, Larson MG, et al. Low-densitylipoprotein cholesterol concentrations and risk of incident diabetes: epidemiological and genetic insights from the Framingham Heart Study. Diabetologia 2015;58:2774-80.

43. Besseling J, Kastelein JJ, Defesche JC, et al. Association between familial hypercholesterolemia and prevalence of type 2 diabetes mellitus. JAMA 2015;313:1029-36.

44. Janghorbani M, Soltanian N, Amini M, et al. Low-density lipoprotein cholesterol and risk of type 2 diabetes: The Isfahan diabetes prevention study. Diabetes Metab Syndr 2018;12:715-9.

45. Walker RJ, Strom Williams J, Egede LE. Influence of Race, Ethnicity and Social Determinants of Health on Diabetes Outcomes. Am J Med Sci 2016;351:366-73.

46. Song Q, Liu X, Wang A, et al. Associations between nontraditional lipid measures and risk for type 2 diabetes mellitus in a Chinese community population: a crosssectional study. Lipids Health Dis 2016;15:70.

47. Pan $W$, Sun $W$, Yang S, et al. LDL-C plays a causal role on T2DM: a Mendelian randomization analysis. Aging (Albany NY) 2020;12:2584-94.

48. Pollock BD, Chen W, Harville EW, et al. Differential sex effects of systolic blood pressure and low-density lipoprotein cholesterol on type 2 diabetes: Life course data from the Bogalusa Heart Study. J Diabetes 2018;10:449-57.

49. Zhang X, Ji L, Ran X, et al. Gender disparities in lipid goal attainment among type 2 diabetes outpatients with coronary heart disease: results from the CCMR-3B study. Sci Rep 2017;7:12648.

50. Billimek J, Malik S, Sorkin DH, et al. Understanding disparities in lipid management among patients with type 2 diabetes: gender differences in medication nonadherence after treatment intensification. Womens Health Issues 2015;25:6-12. 


\section{Supplementary}

Table S1 Cutting off values of the tertiles of LDL-c/HDL-c, TG/HDL-c, TC/HDL-c ratios in the cross-sectional survey in Shanghai

\begin{tabular}{lccccccc}
\multirow{2}{*}{$\begin{array}{l}\text { Cutting off } \\
\text { value }\end{array}$} & \multicolumn{3}{c}{ Male } & & \multicolumn{2}{c}{ Female } \\
\cline { 2 - 3 } & LDL-c/HDL-c ratio & TG/HDL-c ratio & TC/HDL-c ratio & & LDL-c/HDL-c ratio & TG/HDL-c ratio & TC/HDL-C ratio \\
\hline $1^{\text {st }}$ tertile & 2.05 & 0.79 & 3.41 & 1.89 & 0.66 & 3.15 \\
$2^{\text {nd }}$ tertile & 2.88 & 1.39 & 4.38 & 2.62 & 1.15 & 4.07 \\
\hline
\end{tabular}

LDL-c, low-density lipoprotein cholesterol; TG, Triglyceride; HDL-c, high-density lipoprotein cholesterol; TC, total cholesterol. 